\title{
DE LAAPLICACIÓN A LA PARTICIPACIÓN ACTIVA DE LAS TIC EN EDUCACIÓN INFANTIL
}

\section{OF THE 'APPLICATION' TO 'ACTIVE PARTICIPATION' THE ICT IN EARLY CHILDHOOD EDUCATION}

\author{
Dr. John Siraj-Blatchford ${ }^{1}$ \\ johnsb@gmx.com \\ Dra. Rosalía Romero Tena ${ }^{2}$ \\ rromero@us.es \\ (1) University of Plymouth, School of Education, \\ Devon, United Kigdom \\ (2) Universidad de Sevilla-España \\ Facultad de Ciencias de la Educación.. Dpto de Didácticas y Organización Educativa \\ c/ Pirotecnia s/n. Sevilla-España
}

Resumen: En el artículo se recogen datos sobre cómo se han incorporado las tecnologías en los hogares y cómo dicha inclusión ha repercutido en el uso cada vez mayor de niños y niñas en edades más tempranas. Panorama que plantea una línea de estudio que parte de las teorias de Piaget y Vigotsky en las se aprovecha el juego digital como estrategia de aprendizaje para los pequeños y en la que el adulto es el "andamiaje" fundamental. Muestra la importancia del ambiente positivo familiar y escolar en el uso de las tecnologías para que la ZPF promueva la motivación en el niño y maximice su experiencia.

Palabras clave: Tecnología, Infantil, ZDP, juego digital, familia.

Abstract: This paper discuss about how technology has been incorporated into the home and how the said technologies has impacto on its higher use by childerns. This scenario set a study line based on Piaget and Vigotsky theories, where digital game scheme take advance as a lerning strategy for the children and where adults became the critical scaffolding. Shows the importance a of positive family and school environment in the use of technologies in the way that ZDP promotes a higher motivation in the child and maximizes their experience. Keywords: Technology, child, ZDP, digital game, family.

$$
-165-
$$

Píxel-Bit. Revista de Medios y Educación. No 51 Julio 2017. ISSN: 1133-8482. e-ISSN: 2171-7966. doi: http://dx.doi.org/10.12795/pixelbit.2017.i51.11 


\section{SOCIEDAD DIGITAL E INFANCIA.}

Las tecnologías han penetrado profundamente en todos los hogares, como así se ha venido mostrando en recientes estudios e informes, como el realizado por el Observatorio Nacional de las Telecomunicaciones y de la Sociedad de la Información(ONTSI-2014) sobre la evolución de las TIC en los hogares españoles durante los últimos 13 años. O el del Instituto Nacional de Estadística (INE del 3 de octubre 2016) sobre el uso de las tecnologías en el hogar. Concretamente, este último, tras realizar una encuesta sobre Equipamiento y uso de Tecnologías de la Información y Comunicación en los Hogares, destaca que el 99,3\% de los hogares dispone de teléfono (fijo o móvil) y el 75,9\% tiene ambos tipos de terminales y que el $80,1 \%$ de las viviendas el principal tipo de conexión a Internet es por banda ancha. Los que no disponen de ellas señalan como principales razones, bien que tienen pocos conocimientos para utilizarlo $(41,3 \%)$, o bien porque los costes del equipo son demasiado altos $(25,6 \%)$; o porque los costes de conexión resultan demasiado elevados (20,7\%). (INE, 2016)

Estos hallazgos ponen de manifiesto algunos datos relevantes relacionados con el camino que está tomando la evolución del uso de internet y del ordenador en los más pequeños. Esta evolución refleja un aumento significativo del uso en niños de edades inferiores a los 10 años y coincide, además, con un incremento en cuanto a la disponibilidad de teléfono móvil a partir de los 8-10 años hasta alcanzar el $93,9 \%$ en la población de 15 años. (INE, 2016)

Se completan estos datos con otros procedentes del Estudio General de Medios (EGM, 2012) realizado por la Asociación para la investigación de medios de comunicación, cuyo objetivo fue conocer el uso del ordenador y acceso a internet en niños de entre 4 y 13 años en sus hogares. Encontrando que en al menos 2.000 hogares de $\operatorname{los} 2.728$ de los que se ha recogido información, había un niño/a de esta franja de edad que utilizaba el ordenador. En estos gráficos se ve el aumento del uso del ordenador con relación a la edad (figura 1) y el lugar de uso o acceso (figura 2).

Estos estudios muestran datos sobre cómo los niños cada vez más temprano comienzan a usar el ordenador e internet. Padres y educadores tendríamos que pensar en este acelerado incremento y preocuparnos por controlar esta inmersión progresiva en el mundo tecnológico.

Entre las principales razones, podríamos señalar, la falta de habilidades técnicas, críticas y sociales que tienen nuestros pequeños y que suponen un riesgo para ellos y para los que los rodean. Como veremos a lo largo del artículo se está buscando, desde los hogares y desde los centros educativos, la mejor manera de hacer que nuestros niños y niñas crezcan siendo participantes y productores de las tecnologías que les rodean.

En el hogar, la tablet ha comenzado a posicionarse como una nueva pantalla de referencia para las familias, compartiendo espacio con una TV evolucionada (ahora Smart TV), el smartphone y el ordenador. No existen datos específicos en España más allá de la presencia global de este dispositivo electrónico en los hogares (un 28\% a finales del año 2014, según el ONTSI), pero sí en otras regiones europeas, como en Reino Unido, donde estudios como el de Ofcom (2013), que indica que 1 de cada 3 niños tiene acceso a una tableta (hasta el $43 \%$ de los niños

- 166 -

Píxel-Bit. Revista de Medios y Educación. No 51 Julio 2017. ISSN: 1133-8482. e-ISSN: 2171-7966. doi: http://dx.doi.org/10.12795/pixelbit.2017.i51.11 


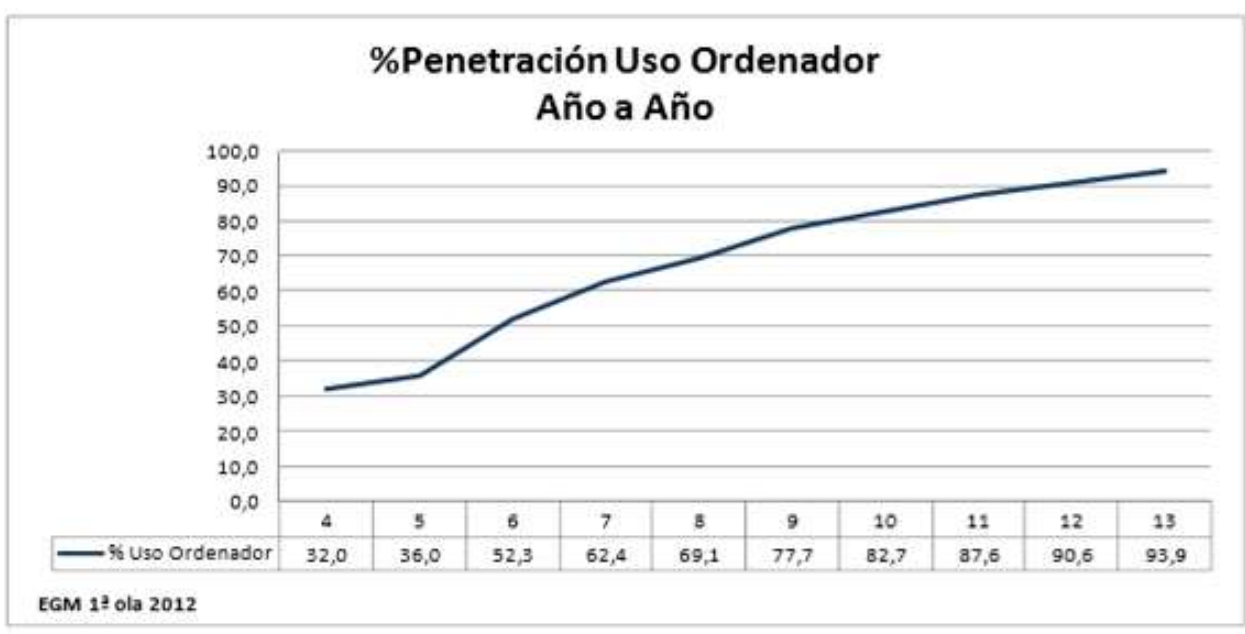

Figura 1.- Uso del ordenador en función de la edad. Extraído de EGM $1^{a}$ ola 2012

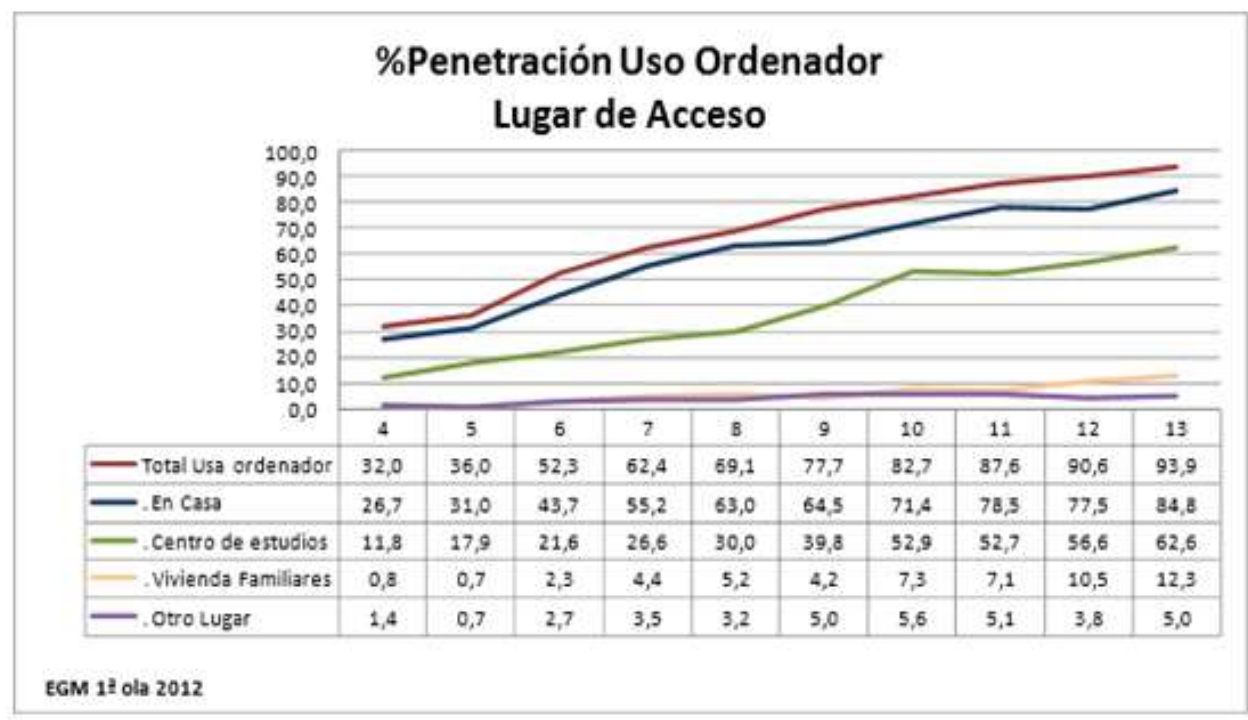

Figura 2.- Lugar donde suele utilizar el ordenador. Extraído de EGM $1^{a}$ ola 2012

$-167-$

Píxel-Bit. Revista de Medios y Educación. $N^{o} 51$ Julio 2017. ISSN: 1133-8482. PIXEL e-ISSN: 2171-7966. doi: http://dx.doi.org/10.12795/pixelbit.2017.i51.11 
de entre 5-15 años y el 11\% de los niños de entre 3-4 años), llegando, incluso, a preferir su uso antes que ver la televisión. Más allá de Europa, una encuesta americana ha puesto de manifiesto que, en el año 2014, el 59\% de los hogares en EE.UU. con niños y niñas de 2 a 12 años disponían de una tablet para jugar (NPD Group, 2014).

Tomando como reflexión el informe «Young Children (0-8) and digital technology: A qualitative exploratory study across seven countries», ofrecido por el Departamento de Proyectos Europeos del Instituto Nacional de Tecnologías Educativas y de Formación del Profesorado (INTEF-2016) proyecto pionero en Europa por realizarse con niños de edades de 0-8 años, cuyo objetivos son conocer cómo los niños/as usan las tecnologías (incluyendo experiencias que tienen los niños y sus familias con las tecnologías) y cómo sus familias intervienen en este uso, e identificar los beneficios y los riesgos potenciales asociados con las interacciones que los niños hacen con ellas. Extraemos que las tablets, cuando están disponibles, son los dispositivos preferidos por los niños, por el tamaño de sus pantallas $\mathrm{y}$, sobre todo, por su multifuncionalidad. Una tableta es más pequeña y mucho más cómoda que el ordenador de sobremesa, pero ofrece las mismas oportunidades para los niños y tiene muchas más opciones que el teléfono móvil. La mayoría de los niños y niñas usan las tabletas para jugar y ver dibujos animados, pero a algunos les gusta crear cosas con ellas, dibujar, hacer fotos y recopilar vídeos. $\mathrm{La}$ portabilidad de las tabletas posibilita el poder llevárselas a otra habitación, a un largo viaje o a cualquier sitio. Desgraciadamente muchos padres las usan a menudo cuando necesitan que sus hijos estén en silencio y ocupados en momentos de largas esperas. (INTEF, 2016:6)

Otros de los medios electrónicos más usados por nuestros pequeños son los teléfonos inteligentes ya que permiten a los niños ver vídeos, jugar, enviar mensajes, hacer fotos y llevar a cabo vídeo llamadas. En la mayoría de los casos, los niños usan los dispositivos de sus padres en diferentes contextos y para distintas actividades, sobre todo para pasar ratos a lo largo del día, para estar ocupados durante un tiempo de espera o cuando los padres necesitan algo de tiempo para ellos mismos. En este contexto, cuando los niños recurren a los teléfonos inteligentes de sus padres están haciendo uso de un dispositivo que no está configurado para ellos y que normalmente se conecta de manera automática a una red Wi-Fi, si la hay disponible. El número de niños que posee su propio teléfono inteligente varía mucho de unos países a otros, pero sí que hay un gran número de ellos en niños y niñas de menos de 8 años, eso sí, no todos con acceso a Internet (INTEF, 2016, P7).

Vandewater et al. (2007) realizó un estudio en Estados Unidos para describir el acceso al uso de los medios tecnológicos en niños de entre 0-6 años, mostró que, en un día típico, el $75 \%$ ve televisión y el $32 \%$ videos, por aproximadamente un promedio de $1 \mathrm{~h} .20^{\prime}$ diaria. Además, el $27 \%$ de los niños entre los 5 y 6 años usan el ordenador casi 1h. diaria. En relación con la disponibilidad de la televisión, se encontró que una $1 / 5$ parte de los niños de 0 a 2 y $1 / 3$ de los de 5 a 6 años, tienen televisor en su habitación. La mayoría de los niños de 3 a 6 años cumplen con la recomendación de no ver televisión más $2 \mathrm{~h}$. diarias, pero el $70 \%$ de entre 0 y 2 años no la cumplen.

$$
-168-
$$


Camargo y Orozco (2013) muestran hallazgos muy interesantes que confirman los datos presentados anteriormente, y añaden que no sólo se dispone de medios y dispositivos electrónicos en el hogar, sino también, en la habitación de los niños. En particular, se dispone de televisor con acceso a transmisión por cable, computador e internet, así como consolas, videojuegos manuales y teléfonos móviles. Esto se va incrementado con la edad y en el sexo masculino. Sugieren que la mejora en los ingresos familiares es un factor asociado a la disponibilidad de medios electrónicos en el hogar y, más concretamente, en la disponibilidad en la habitación. (p.181)

Vandewater et al. (2007), encontraron factores asociados al exceso de visionado de la televisión, (no exceda las $2 \mathrm{~h}$. diarias en niños entre los 0 y 6 años), concretamente a una variable significativa como la estructura familiar, categorizada como niños que viven con un solo padre, con la madre o con ambos, así como con disponer de televisor en el cuarto del niño o televisor en el hogar (p.182)

Los efectos de ver la televisión más de $2 \mathrm{~h}$. diarias sobre las destrezas sociales y el comportamiento de los niños a la edad de entre 2-5 años, fueron analizados por Mistry et al. (2007), quienes encontraron que la exposición sostenida entre los $2 \frac{1}{2}$ años y los 5 años se asocia con problemas de sueño y de atención, así como con comportamientos agresivos y falta de interacción social.

Para estos estudios se establecieron como puntos de corte, ver televisión más de $2 \mathrm{~h}$./ dia, y para el uso de computador y videojuegos más de 1h./día, recomendaciones sobre actividad física $\mathrm{y}$ comportamiento sedentarios establecidas internacionalmente por la American Academy of Pediatrics, la National Association for Sport and Physical Education (NASPE) y el
Department of Health and Aging (DoHA) del gobierno australiano.

Es importante considerar la marcada diferencia entre las generaciones actuales de niños en su primera infancia, comparados con los niños de generaciones precedentes, pues la tecnología son parte de su vida desde el nacimiento, hecho que debe aprender a manejarse, tanto por los padres como por las instituciones educativas, con el fin de utilizarlos positivamente para contribuir a la educación de las nuevas generaciones y disminuir los potenciales efectos negativos que a la fecha han sido planteados (Camargo \& Orozco, 2013), p.184.

Pero lo cierto es que la edad de inicio en su uso es mucho más temprana. Los niños de 2 y 3 años de edad acceden de forma habitual a los terminales de sus padres, manejando diversas aplicaciones principalmente juegos, aplicaciones para pintar o colorear y cadenas de televisión que ofrecen series infantiles a través de estos dispositivos. Algunos de estos niños ya recorren Youtube saltando de vídeo en vídeo, o repasan de una en una las fotografías que sus padres tienen en sus galerías de fotos. (Canovas, 2014), p.13

En esta línea, el estudio realizado por Marques (2014), resalta el impacto positivo de su uso en la escuela para que los niños y niñas mejoren su comprensión, creatividad, capacidad de memoria, motivación para aprender, desarrollo de competencias digitales y el aprendizaje autónomo. En esta misma línea, también se posiciona el estudio realizado por Cánovas (2014), en el que se afirma que los niños y niñas deben iniciarse lo antes posible en el manejo de una tableta, pero:

...siempre y cuando esto suceda con el acompañamiento de sus padres y éstos estén

- 169 -

Píxel-Bit. Revista de Medios y Educación. No 51 Julio 2017. ISSN: 1133-8482. e-ISSN: 2171-7966. doi: http://dx.doi.org/10.12795/pixelbit.2017.i51.11 
decididamente implicados en su formación. No se trata sólo de estar delante, en muchas ocasiones hay que interactuar y compartir las primeras experiencias con ellos. (pp. 3 4):

\section{JUEGO INFANTIL CON TECNOLO- GÍAS. Del ZPD al ZPF.}

Ya a finales de siglo XX había estudios que manifestaban que los niños que utilizan ordenadores en edad preescolar lograban mejores resultados en las medidas de la metacognición, concretamente eran más capaces de tener en cuenta una serie de diferentes estados mentales de forma simultánea y tenían teorías más sofisticadas que los que no los usaban. (Fletcher-Flinn \& Suddendorf, 1996),

En esta misma línea otros estudios (Falloon \& Khoo, 2014; Romero, 2011; Siraj-Blatchford \& Morgan, 2006; Sung et al, 2015) nos muestran las bondades de la tecnologías así como las diferentes formas de usarlas jugando con las TIC, son experiencias que ayudan a los niños pequeños a desarrollarse. Las tecnologías son las más adecuada para animar a una gran cantidad de juego con un propósito concreto y siempre con una finalidad exploratoria. Entre otras cosas porque a través de ellas se puede fomentar la discusión, la creatividad, la resolución de problemas, la toma de riesgos y desarrollan un pensamiento más flexible. Todo esto se puede lograr en un ambiente de juego centrado y sensible. Los niños están aprendiendo sobre el mundo real y la exploración a través del juego (Figura 3 ). Se desarrollan como comunicadores y colaboradores, teniendo en cuenta las necesidades de los demás a medida que trabajan juntos. Desarrollan su uso del lenguaje en su juego y con sus historias. Se desarrollan como pensadores creativos.

Centrándonos en la importancia del juego como estrategia de aprendizaje por excelencia en estas edades, retomamos algunas ideas de Piaget (1969) y Vygotsky (1978). De Piaget su afirmación de que los niños dan sentido a las cosas principalmente a través de sus acciones en su entorno. Y de Vygotsky el valor de la cultura y el contexto social, que sirve de guía y les ayuda en el proceso de aprendizaje. Ambos tomaron la idea de que las operaciones complejas cognitivas eran emergentes y eran conseguidas como resultados de un aprendizaje significativo previo más que las suma de sus partes.

En el caso de la lectura, las bases para el aprendizaje se establecen en la representación del el juego, en la manipulación de objetos simbólicos, ya que en un primer momento permiten la trasformación de un objetos por otro, y luego en las interacciones lúdicas del niño con la cultura impresa y el medio ambiente.

Como Davis y Tall (2015) explican tomando el ejemplo de un niño que está aprendiendo a contar:

Nuestro cerebro toma como base para la reflexión la memoria de experiencias previas, y así como nosotros categorizamos percepciones visuales de cosas que se parecen a una taza en categorías de taza así categorizamos patrones de acción como los movimientos dirigidos con interacciones estandarizadas sincronizadas con la categoría de contar. (p.4)

Para Vygotsky (1978), este desarrollo del niño es fundamental que sea apoyado por un adulto que le sirva de «andamiaje» para conseguir los logros cognitivos dentro de la «zona de desarrollo próximo»(ZDP), refiriéndose a:

$$
-170 \text { - }
$$




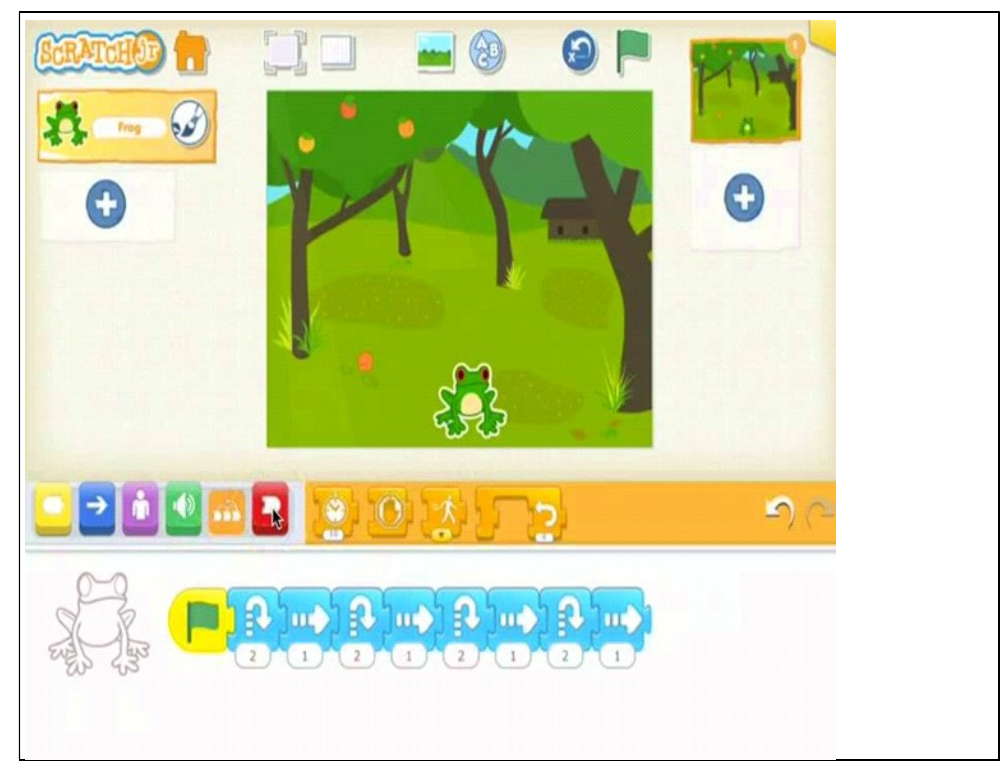

Figura 3.- Captura pantalla Scratch Jr. para niños de Infantil

la distancia entre el nivel real de desarrollo, determinado por la capacidad de resolver independientemente un problema, y el nivel de desarrollo potencial, determinado a través de la resolución de un problema bajo la guía de un adulto o en colaboración con otro compañero más capaz. (p.133).

Se entiende, usualmente, por «andamiaje» (Figura 4) una situación de interacción entre un sujeto experto, o más experimentado en un dominio, y otro novato, o menos experto, en la que el formato

de la interacción tiene por objetivo que el sujeto menos experto se apropie gradualmente del saber experto; el formato debería contemplar que el novato participe desde el comienzo en una tarea reconocidamente compleja, aunque su participación inicial sea sobre aspectos parciales o locales de la actividad global y aun cuando se requiera del «andamiaje» del sujeto más experto para poder resolverse. La idea de «andamiaje» se refiere, por tanto, a que la actividad se resuelve «colaborativamente» teniendo en el inicio un control mayor o casi total de ella el sujeto experto, pero delegándolo gradualmente sobre el novato. La estructura de andamiaje alude a un tipo de ayuda que debe tener como requisito su propio desmontaje progresivo.

Recuérdese, en primera instancia, como afirmáramos al tratar los procesos de adquisición de la escritura, que el juego es, ante todo, una de las principales, o incluso, la principal actividad del niño. Con esto Vygotsky señala el carácter central del juego en la vida del niño, además el juego parece estar caracterizado como una de las maneras de participar el niño en la cultura, es su actividad cultural típica, como lo será luego, de adulto el trabajo. Es decir, según esta 


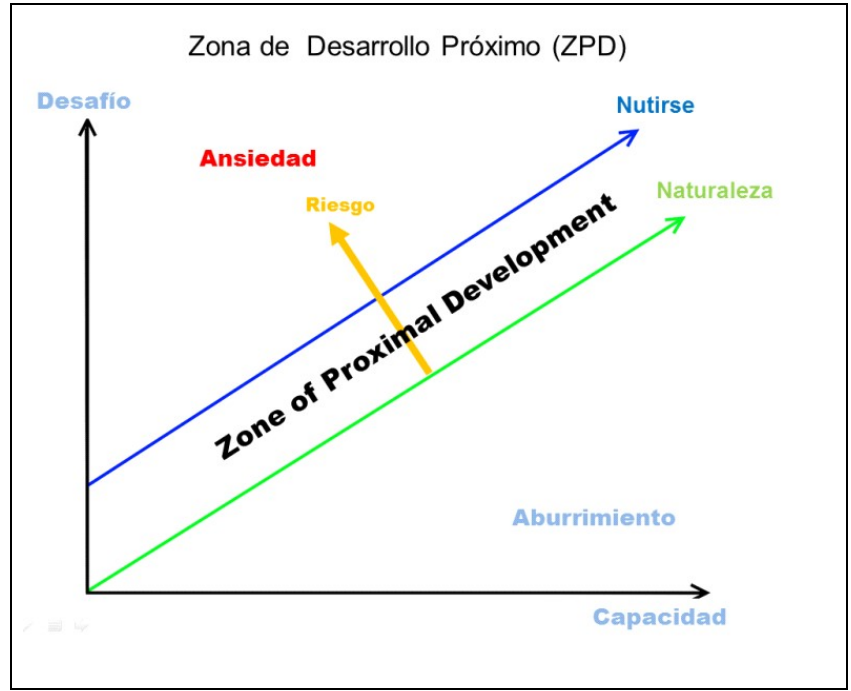

Figura 4.- ZDP según Vygotsky.

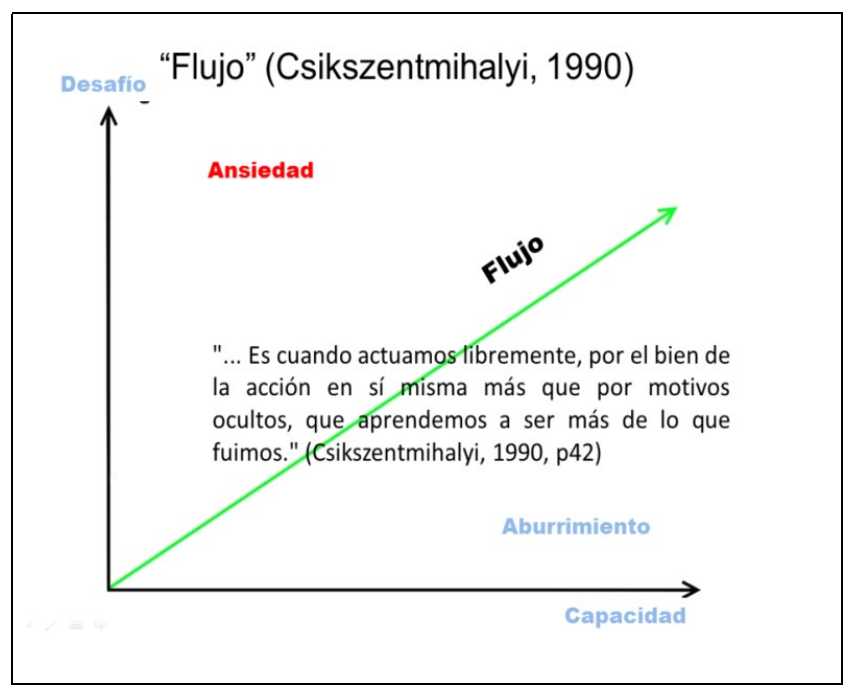

Figura 5.- Flujo de Csikszentmihalyi.

$$
-172-
$$

PIXEL Píxel-Bit. Revista de Medios y Educación. No 51 Julio 2017. ISSN: 1133-8482. e-ISSN: 2171-7966. doi: http://dx.doi.org/10.12795/pixelbit.2017.i51.11 
perspectiva, el juego resulta una actividad cultural. Seamos más precisos, el juego que interesa a efectos de ponderar el desarrollo del niño en términos de su apropiación de los instrumentos de la cultura, es un juego regulado más o menos por la cultura misma. Esto no agota los sentidos posibles y la variedad de formas del juego, sólo pretende acotar en cuáles de esos sentidos o variedades el juego protagoniza un rol central en el desarrollo del niño, en qué condiciones o cuáles de sus procesos implican la creación de Zonas de Desarrollo Próximo.

Algunas aclaraciones, la primera no toda actividad lúdica genera ZDP (del mismo modo en que no todo aprendizaje ni enseñanza lo hacen) y segundo todo juego comporta la instalación de una situación imaginaria y la sujeción a ciertas reglas de conducta («reglas de juego»al fin).

En esta línea Siraj-Blatchford y Palmer, (2011) realizaron un estudio para comprobar el aprendizaje de niños de preescolar usando Alphablocks, se identificó la escasez de la formación y capacidad de los educadores de la primera infancia para enseñar fonética adecuada. En el entorno de juego digital de AlphBlocks se encontró que proporcionó «andamiaje» tanto para el adulto como para los niños:

Se encontró que mientras que el investigador y practicante trabajaron junto al niño para facilitar el diálogo y las interacciones de pantalla, se obtuvieron un acuerdo de voluntades, donde el aprendizaje posterior se estaba produciendo en ambos lado. (p. 12)

Otros estudios realizados (Siraj-Blatchford \& Whitebread, 2003; Siraj-Blatchford \& SirajBlatchford, 2006; Siraj-Blatchford \& Morgan, 2009, Sung et al, 2015) han encontrado que los pequeños que están enganchados a los juegos digitales más que en otros juegos tradicionales.

Csikszentmihalyi (1990) describió la completa inmersión, implicación y sentido de satisfacción de los niños cuando juegan con los juegos digitales, esto conducía al sujeto a la llamada «Situación de 'Flujo'», o sea, a un estado en el cual el niño se halla tan involucrado en la tarea que nada le parece más importante (Figura 5).

La situación de flujo constituye una experiencia intrínsecamente placentera que la persona intenta repetir, siempre que le sea posible, para volver a experimentar o sentir esa sensación. En palabras del propio Csikszentmihalyi (1990).

las actividades que llevan al «flujo» son situaciones en las que una persona puede emplear libremente la atención para alcanzar sus objetivos, pues no hay ningún desorden que corregir ni ninguna amenaza para la personalidad de la que haya que defenderse. Hemos llamado a este estado, experiencia de flujo. (p.230),

Laevers y Heylen (2003) subraya que esta inmersión solo puede ocurrir en la zona actividad que conecta con las capacidades del niño, su zona de desarrollo próximo ZDP.

... la unidad de exploración, tiene la necesidad de obtener un mejor contacto con la realidad, el interés intrínseco en cómo las cosas y las personas son, las ganas de experimentar y averiguar. (p.15).

Basawapatna et al (2010) han confirmado la importancia de la obra de Csikszentmihalyi (1990) en la educación en general, y han aplicado un "patrón de análisis del pensamiento computacional» para

proporcionar a sus estudiantes retos progresivos a través de un «suave progreso de proyectos cada vez más complejos». $\mathrm{Al}$ combinar el concepto psicológico de flujo de

$-173-$

Píxel-Bit. Revista de Medios y Educación. No 51 Julio 2017. ISSN: 1133-8482. e-ISSN: 2171-7966. doi: http://dx.doi.org/10.12795/pixelbit.2017.i51.11 


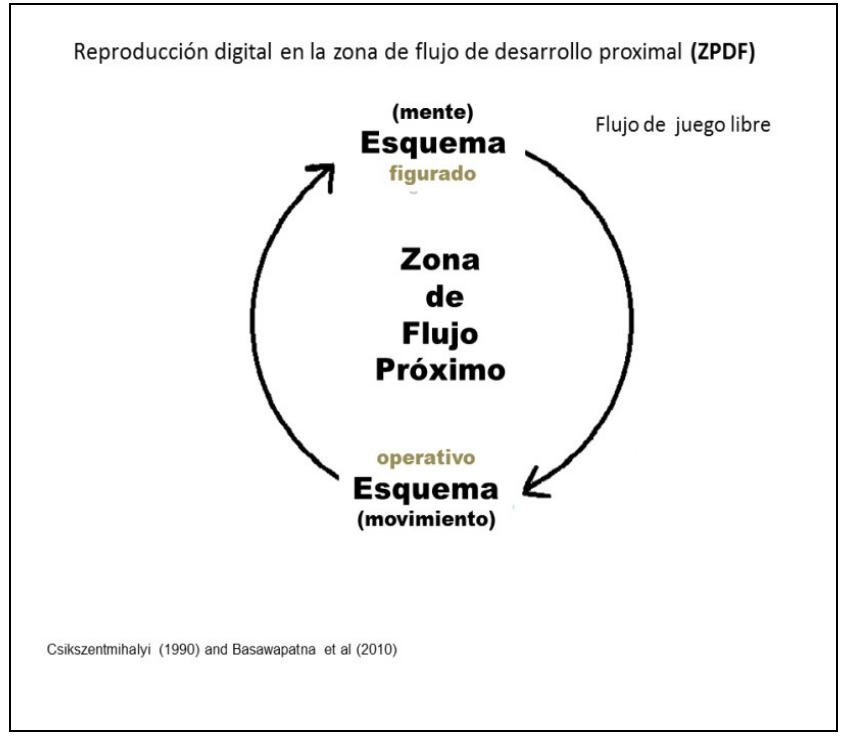

Figura 6.- Zona de Flujo Próximo.

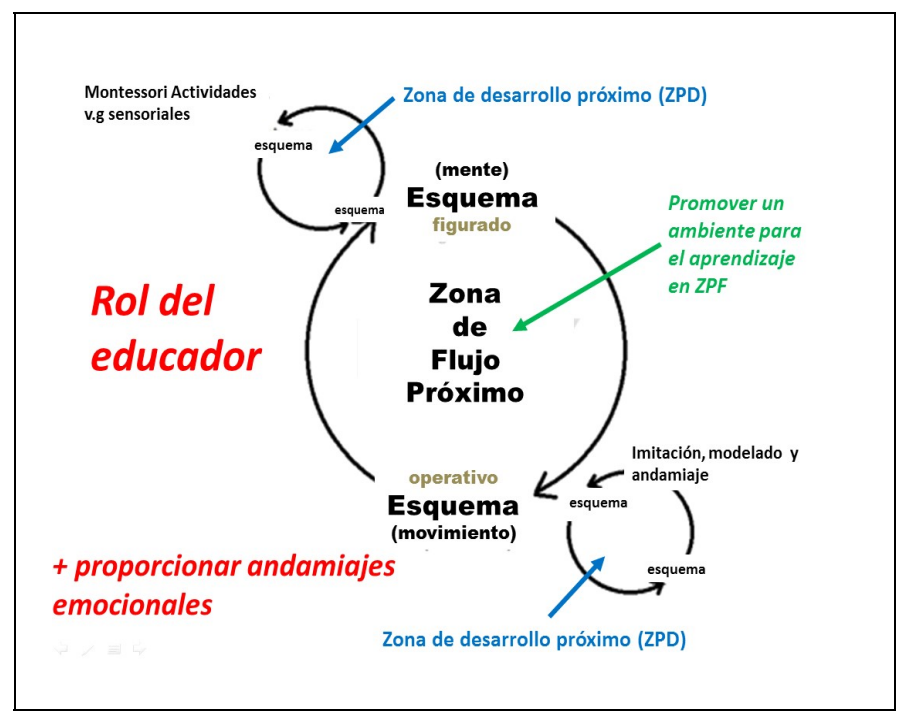

Figura 7.- Zona de Flujo Próximo y el andamiaje adicional.

$$
-174-
$$

Píxel-Bit. Revista de Medios y Educación. $N^{o} 51$ Julio 2017. ISSN: 1133-8482. e-ISSN: 2171-7966. doi: http://dx.doi.org/10.12795/pixelbit.2017.i51.11 
Csikszentmihalyi (1990), con la noción Vygotsky (1969) socio-cultural de la ZDP, Basawapatne et al (2010) hablan de una zona

Optima de flujo proximal (ZPF), que promueve la motivación intrínseca de los niños y maximiza su experiencia de aprendizaje (Figura 6).

Este concepto de la ZPF puede igualmente ser aplicado en el caso del juego. Cuando el juego del niños está sostenido por su propia voluntad de aprender y experimentan por sí mismo es más productivo que cuando hay una instrucción dirigida. Esto constituye un proceso de auto-andamiaje que ya fue identificado en la investigación de Tharp y Gallimore (1991).

Este modelo de análisis que hasta ahora hemos desarrollado sobre la zona de flujo próxima, define el espacio de oportunidad del juego libre que se activa cuando el niño es capaz de abrirse a los nuevos esquemas. Aplicando el concepto cíclico de los procesos de aprendizaje, nosotros tenemos que darnos cuenta de que este «andamiaje» interno incluye esquemas operacionales y figurativos, y el aprendizaje se lleva a cabo a través de una relación recurrente entre las acciones del niño en el mundo físico y sus entendimientos novedoso de estas acciones. Como podemos ver en la figura fuera del ZPF, las funciones cognitivas aplicadas en el juego pueden desarrollarse a través de actividades de aprendizaje enfocadas que pueden ser sostenidas deliberadamente por el adulto, o ser simplemente una imitación de la conducta que el niño ha observado. (Siraj-Blatchford y Brock, 2015)

Desde esta perspectiva, la tarea del educador en la supervisión del flujo libre dentro de la ZPF será la de permitir acceso a materiales y entornos y, a través de la modelización o andamiaje adicional, con independencia de lo que diga el niño tiene que mantenerse dentro del ZPF (Figura 7).

Investigaciones realizadas proporcionan una gran cantidad de ejemplos sobre el uso de juegos

digitales en los hogares acompañados de padres o de los educadores. Por ejemplo, la investigación llevada a cabo por Falloon y Khoo (2014) sobre Puppet Pals HD que se utiliza para apoyar la narración de historias; Pic Collage, para resumir el aprendizaje de una unidad de celebraciones; y Popplet para el desarrollo de plan de historia, muestran la importancia de apoyo del adulto. Una amplia gama de ejemplos ofrecidos por SirajBlatchford y Morgan (2006) y Sung et al (2015) muestran cómo los padres y los niños pueden aprender diversas habilidades de alfabetización digital con respecto a la otra; cómo navegar aplicaciones particulares, cómo manipular los juguetes programables (Figura 8 y9).

En todos estos juegos digitales Yelland y Masters (2007) identificaron la necesidad de un andamiaje cognitivo, afectivo y técnico para ayudar a mantener a los pequeños en la tarea y animar seguir a los niveles superiores de pensamiento.

En este sentido Stephen (2010) ha resaltado dos grandes ideas relacionadas con la pedagogía en la primera infancia una centrada en la libertad de elección de la actividad por parte del niño, y la otra en el desarrollo a través del juego. Argumentando que es necesario un cierto grado de iniciación de la actividad por parte de un adulto para conseguir aprendizaje (p.18).

Investigadores, educadores y padres, estamos convencidos que el primer acercamiento a las tecnologías es en el hogar comienzan a edades muy tempranas, esta situación debe hacernos reflexionar sobre

$-175-$

Píxel-Bit. Revista de Medios y Educación. No 51 Julio 2017. ISSN: 1133-8482. e-ISSN: 2171-7966. doi: http://dx.doi.org/10.12795/pixelbit.2017.i51.11 


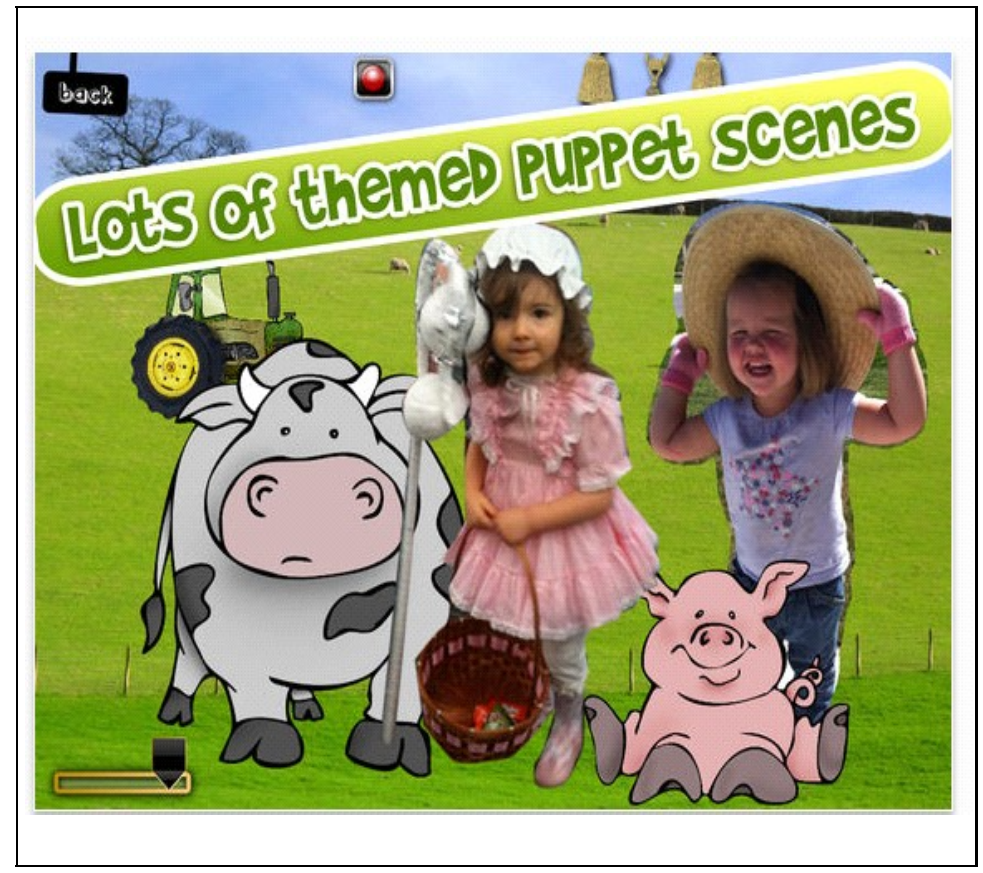

Figura 8.-. Puppet Pals HD

cómo hacer las cosas para educar a nuestros niños y niñas en cómo ser usuarios de las tecnologías sin riesgo y cómo aprovechar el juego digital como actividad para el aprendizaje. Creando buenos hábitos de uso, y haciéndoles participantes y productores de las mismas. Aunque hay cierta confusión con respecto a los objetivos educativos de las TIC en la educación infantil, en su mayoría consecuencia de investigaciones que argumentan ideas contradictorias, la tendencia actual y la más realista es aceptar que están ahí, y educar teniéndolas presentes para sacar partido a sus bondades.

Las TIC están siendo utilizadas como instrumento político del discurso de innovación pero no se arbitran medidas para que estas tengan lugar. Por eso, se adaptan a la cultura vigente del centro educativo y a los contenidos y los métodos de enseñanza que son habituales en ellos. (Montero, 2010:315)

De esta forma en el Reino Unido la política de proyectos Effective Provision for Preschool Education (EPPE) controlan el progreso de 3.000 pequeños desde $1999 \mathrm{y}$ han encontrado que los pequeños con un ambiente de estudio hogareño positivo de aprendizaje consiguen mejoras desde los primeros años y durante toda escolarización. Mientras que niños con un HLE (ambiente hogareño de aprendizaje) pobre ya tenían desventajas a los tres años en las calificaciones al entrar en preescolar (Sylva et al 2006).

Aunque EPPE encontró una relación entre la calidad del HLE y el status socioeconómico -SES- (status socioeconómico) mostró que en relación a la clase social la familia suelen mejorar los resultados de los niños a partir de

$$
\text { - } 176 \text { - }
$$

Píxel-Bit. Revista de Medios y Educación. No 51 Julio 2017. ISSN: 1133-8482. e-ISSN: 2171-7966. doi: http://dx.doi.org/10.12795/pixelbit.2017.i51.11 


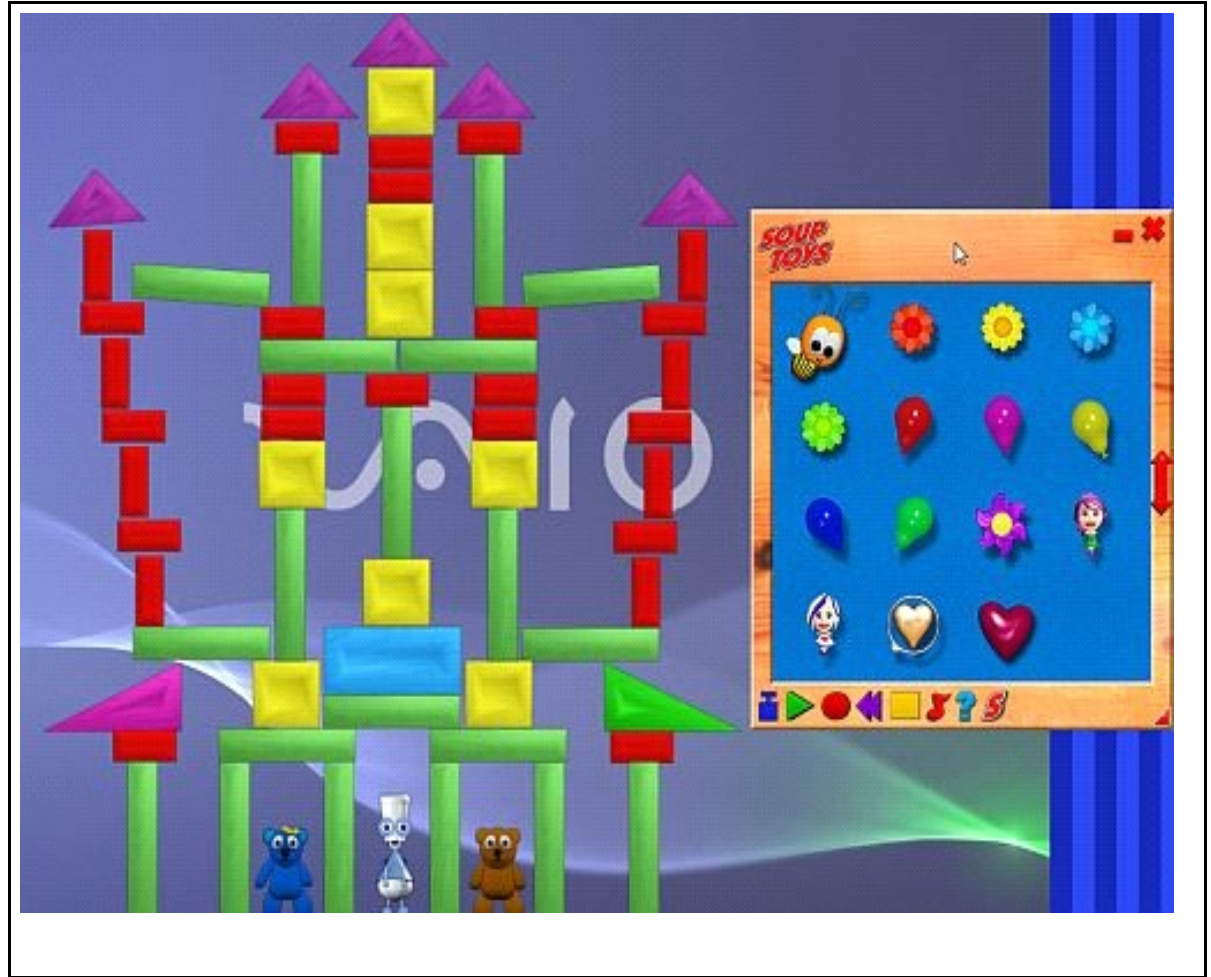

Figura 9.-. Ejemplo de juego lógico.

los que hicieron con los niños a través de un esfuerzo temprano en el ambiente de aprendizaje familiar hogareño. Un buen ambiente de familiar promueve los buenos resultados de los pequeños y eso tiene un efecto independiente de la clase social.

Lo que los padres hacen es de vital importancia y puede contrarrestar otras influencias desventajosas, concretamente durante el periodo de preescolar, por esta razón el entorno en preescolar que no favorecen un apoyo familiar y positivo HLEs se supone que no tienen en cuenta un elemento muy importante para aumentar el desarrollo socio-comportamental a largo plazo (Sylva et al 2006:54)
El resultados de los proyecto EPPE han mostrado que muchos padres y comunidades desfavorecidas ayuda a los pequeños a superar los estudios (Siraj-Blatchford \& Morgan, 2009, Silva et al., 2004; SirajBlatchford \& Mayo, 2012). Importantes investigaciones están disponibles y dan resultados de los beneficios que tiene la ayuda o guía de los padres en el uso ordenador.

En España el estudio realizado por Lera (2007) en el que se evalúa la integración de las tecnologías en aulas de educación infantil, muestra a un profesorado que integra el ordenador en su práctica educativa con dificultades. Estos hallazgos tienen su explicación cuando se tiene en cuenta la realidad de las aulas (número de niños por - 177 -

Píxel-Bit. Revista de Medios y Educación. No 51 Julio 2017. ISSN: 1133-8482. e-ISSN: 2171-7966. doi: http://dx.doi.org/10.12795/pixelbit.2017.i51.11 
educador, la formación del profesorado en TIC, la calidad del entorno y los métodos educativos que se desarrollan) aspectos que marcan diferencias importantes con otros países europeos.

Se insiste en la correcta utilización de las TIC para mejorar la transmisión del conocimiento y la innovación para llegar a una educación de calidad. Las TIC no constituyen un fin en sí mismo, sino solo un medio adicional para mejorar la calidad y preparar a los alumnos para la sociedad de la información.

En el Real Decreto1630/2006 recoge en el punto 3 del artículo 5 dedicado a contenidos educativos y currículo de la E. Infantil se escribe

Corresponde a las administraciones educativas fomentar una primera aproximación a la lengua extranjera en los aprendizajes del segundo ciclo de la E.I, especialmente el último año. Asimismo, fomentarán una primera aproximación a la lectura y escritura, así como experiencias de iniciación temprana en habilidades numéricas básicas, en las tecnologías de la información y de la comunicación y en ...(p.475)

Con esto se hace patente el compromiso de velar por una educación en y con las tecnologías al servicio del aprendizaje de los estudiantes, pero no esto implica una puesta en escena real en las aulas.

\section{DISCUSIÓN.}

Tras el análisis de documentos e investigaciones sobre el acceso de los más pequeños a la TIC, se puede comprobar la evolución creciente del uso de las TIC por parte de niños y niñas en los hogares a edades cada vez más tempranas. El primer contacto que tienen los niños con las tecnologías, es en casa y en algunas ocasiones en su habitación, esto promueve la falta de la supervisión de un adulto. Estos antecedentes son una gran preocupación que nos impulsan a intentar conocer qué ocurre en estos primeros momentos, cómo los niños usan las tecnologías y qué experiencias tienen con ellas así como el papel de la familia. Buscar los beneficios y conocer los riesgos asociados al uso de las tecnologías es el eje que nos lleva al debate sobre con qué ideas previas y usos nos enfrentamos los docentes cuando los pequeños acceden por primera vez a las aulas de infantil así como buscar y plantear la mejor forma de trabajarlos dentro y fuera de las mismas.

Son estos conocimientos previos que traen los que debemos considerar y tener en cuenta cuando integramos las tecnologías en las aulas de Infantil. En esta línea y partiendo de las teorías de Piaget y Vygotsky junto a la de Csikszentmihalyi (1990), las que nos describen la completa inmersión, implicación y sentido de satisfacción de los niños cuando juegan con los juegos digitales, que conduce al sujeto a la llamada «Situación de 'Flujo», o sea, a un estado en el cual el niño se halla tan involucrado en la tarea que nada le parece más importante que lo que hace en este momento. Pero son Basawapatne et al (2010) combinando las ideas anteriores, los que hablan de una zona optima de flujo proximal (ZPF), que promueve la motivación intrínseca de los niños y maximiza su experiencia de aprendizaje. Desde esta perspectiva el adulto adquiere un papel importante convirtiéndose en el andamiaje que necesita el niño para que el juego genere esas experiencias placenteras y con las que ha de aprender. El juego digital incita a que quiera estar por su propia voluntad aprendiendo y experimentando por

- 178 -

Píxel-Bit. Revista de Medios y Educación. No 51 Julio 2017. ISSN: 1133-8482. e-ISSN: 2171-7966. doi: http://dx.doi.org/10.12795/pixelbit.2017.i51.11 
sí mismo, situación más productiva que una instrucción dirigida.

Esto nos que lleva a plantear líneas de trabajo e investigación sobre la importancia del ambiente familiar y sobre cómo hacer y diseñar propuestas educativas participativas (adulto-niño) para educar a los más pequeños en utilizar las tecnologías sin riesgos, haciéndolos protagonista y constructores de las mismas.

Es imprescindible para ello realizar iniciativas en las escuelas que promuevan el uso de las tecnologías de forma progresiva, proponiendo a los niños y niñas tareas en contextos lúdicos; donde aprendan jugando y sobretodo experimentando, facilitando así la competencia de aprender a aprender a la vez que refuerzan el trabajo colaborativo. Es importante insistir en la formación de padres y profesores en el uso de las TIC, implicados fundamentales en la educación de los más pequeños y elementos de enorme influencia en el uso-abuso de las mismas. Y finalmente, y sin lugar a duda, introducir en el currículum oficial de Educación Infantil una verdadera integración de las tecnologías y que no quede reflejado de forma anecdótica el uso de las Tecnologías de Información y de la Comunicación como ocurre actualmente, que haya un compromiso de transformación considerando el educar en ellas y con ellas.

\section{REFERENCIAS}

Australian Government. Department of Health and Aging (DoHA) (2009). Get up and grow: Healthy eating and physical activity for early childhood. Disponible en http:// www.health. gov.au/internet/main/ $\mathrm{p} \mathrm{u} \mathrm{b} 1$ i s h i n g. n s f / C o n t e n t / EDFEDB588460BCE3CA25762B00232A13/ \$File/gug-directorscoord.pdf
Basawapatna, A., Repenning, A., Kyu, H.K., \& Nickerson, H. (2010). The Zones of Proximal Flow: Guiding students through a space of computational thinking skills and challenges. En ICER '13: International Computing Education Research Conference, August 12-14, San Diego, California, USA.

Camargo, D.M \& Orozco, L.C. (2013). Factores asociados a la disponibilidad y uso de medios electrónicos en niños desde preescolar hasta $4^{\circ}$ grado. Biomédica, 33, 175-185.

Cánovas, G. (Dir.) (2014). Menores de Edad y Conectividad Móvil en España: Tablets y Smartphones. Madrid: Centro de Seguridad en Internet para los Menores en España: PROTEGELES.

Csikszentmihalyi, M. (1990). Flow: The Psychology of Optimal Experience. New York: Harper and Row

Csikszentmihalyi, M. (1992). Flow. The Psychology of Happiness. Rider. London.

Davis, M.M., Gance-Cleveland, B., Hassink, S., Johnson, R., Paradis, G. \& Resnicow K. (2007). Recommendations for prevention of childhood obesity. Pediatrics. 120(Suppl.4). Disponible en http://dx.doi.org/ 10.1542/peds.2007-2329E

Davis, G.E. \& Tall, D.O. What is scheme?. Disponible en https:// homepages.warwick.ac.uk/staff/David.Tall/ pdfs/dot2002d-davis-schemes.pdf

Fletcher-Flinn, C. \& Suddendorf, T. (1996). Do computers affect the mind? Journal of Educational Computing Research, 15(2), 97112.

Instituto Nacional de Estadística (INE). Encuesta sobre Equipamiento y Uso de Tecnologías de Información y Comunicación en los Hogares. 3 de octubre 2016. Disponible http://www.ine.es/prensa/np991.pdf

- 179 -

Píxel-Bit. Revista de Medios y Educación. $N^{o} 51$ Julio 2017. ISSN: 1133-8482. e-ISSN: 2171-7966. doi: http://dx.doi.org/10.12795/pixelbit.2017.i51.11 
Laevers, F. \& Heylen, L. (2003). Involvement of Children and Teacher Style. Leuven: University Press

Lera, M J (2007). Calidad de la Educación Infantil: instrumentos de evaluación. Revista de Educación, 343, 301-323. Disponble en http://www.revistaeducacion.mec.es/re343/ re343_14.pdf

Marquès, P. (2014). Metainvestigación 2013-14. Uso educativo de las tabletas digitales en Educación Infantil. Informe final. Disponible en http://es.slideshare.net/ peremarques/uso-educativo-delas-tabletasdigitales-en-educacin-infantil

Mistry, K.B., Minkovitz, C.S., Strobino, D.M. \& Borzekowski, D.L. (2007). Children's television exposure and behavioural and social outcomes at 5.5 years: Does timing of exposure matters? Pediatrics. 120, 762-769. Disponible en http://dx.doi.org/10.1542/peds. 2006-3573

Montero, Ma L. \& Gewerc, A. (2010). De la innovación deseada a la innovación posible. Escuelas alteradas por las TIC. Revista de currículum y formación del profesorado, 14(1), 303-316.

National Association for Sport and Physical Education. Active start: A statement of physical activity guidelines for children from birth to five years. Reston, VA: NASPE Publications; 2002. Disponible en http:// www.shapeamerica.org/publications/ resources/

NPD Group (2014). Kids' Tablet Play and Household Ownership Increases. Reports NPD. Disponible en https://www.npd.com/ wps/portal/npd/us/news/press-releases/ kidstablet-play-and-household-ownershipincreases-reports-npd

Observatorio Nacional de las Telecomunicaciones de la Sociedad de la Información(ONTSI) (2014). XLIII Oleada del
Panel Hogares «Las TIC en los hogares españoles» (1T/2014). Madrid: Ministerio de Industria, Energía y Turismo de España.

Ofcom (2013). The Comunications Market. Report 2013. Londres: Ofcom.

Piaget, J. (1926). The Language and Thought of the Child. New York: Harcourt, Brace

Piagt, J. (1969). The Mechanisms of Perception. New York: Basic Books

Romero Tena, R. (2011). Posibilidades educativas de los videojuegos y juegos digitales en educación en la etapa Infantil. En V. Marín (coord.), Los videojuegos y los juegos digitales como materiales educativos (pp. 79-107). Síntesis. Madrid.

Romero, R.; Román, P. \& Llorente, $\mathrm{M}^{\mathrm{a}} \mathrm{C}$. (2009). Tecnologías en los entornos de Infantil y Primaria. Madrid: Síntesis

Sawyer, R. (2003). Emergence in creativity \& development. En R. Sawyer,, V. JohnSteiner, S. Moran \& D. Feldman,. Creativity \& Development, 12-60, Oxford: Oxford University Press.

Siraj-Blatchford, J. \& Brock. L. (2015). Early Childhood Digital Play in the Zone of Proximal Flow. Disponible en http:// www.schemaplay.com/Docs/CITEI.pdf

Siraj-Blatchford, I. \& Mayo, A. (2012). Social Class and Educational Inequality: The impact of parents and schools. Cambridge: Cambridge University Press.

Siraj-Blatchford, J. \& Morgan, A. (2009). Using ICT in the Early Years: Parents and Practitioners in Partnership, Practical PreSchool Books

Siraj-Blatchford, J. \& Palmer, N. (2011). Knowledge learning processes and ICT in early Childhood education, He Kupu, 2 (5) October, Disponible en http:// www.hekupu.ac.nz/Journal\%20files/ Is sue $5 \% 20$ October $\% 202011 / \mathrm{Kn}$

$-180-$

Píxel-Bit. Revista de Medios y Educación. No 51 Julio 2017. ISSN: 1133-8482. e-ISSN: 2171-7966. doi: http://dx.doi.org/10.12795/pixelbit.2017.i51.11 
o w le d g e \% 20 learning $\% 20$ proc esses $\% 20$ and $\% 20$ ICT $\% 20$ in $\% 20$ early $\% 20$ child hood\%20education.pdf

Siraj-Blatchford, J. \& Siraj-Blatchford, I. (2006). A guide to developing the ICT curriculum for Early Childhood Education. London:Trentham Books in collaboration with Early Education (The British Association for Early Childhood Education)

Stephen, C. (2010). Pedagogy: the silent partner in early years learning. Early Years, 30(1), 15-28.

Sylva, K., Melhuish, E.C., Sammons, P., Siraj-Blatchford, I. \& Taggart, B. (2004). The Effective Provision of Pre-School Education (EPPE) Project: Final report. London: DfES / University of London, Institute of Education.

Sylva, K., Siraj-Blatchford, I. \& Taggart, B. (2006). Early childhood environmental rating scale-extension (ECERS-E) (2nd ed.). London: Trentham Books.

Sung, H.Y., Siraj-Blatchford, J., \& Kucirkova, N. (2015). Your Guide to Outstanding Early Childhood Practice in ICT. London: Step Forward Publishing.

Tharp, R. and Gallimore, R. (1991). A Theory of Teaching as assisted Performance, in Light, P., Sheldon, S. \& M. Woodhead (Eds.) Learning to Think. Oxford: Routledge.

Vandewater, E.A., Rideout, V.J., Wartella, E.A., Huang, X., Lee, J.H. \& Shim, M. (2007). Digital childhood: Electronic media and technology use among infants, toddlers and preschoolers. Pediatrics, 119 (5) e1006-15. Disponible en http://dx.doi.org/10.1542/ peds.2006-1804 DOI: 10.1542/peds.2006-1804 Vygotsky, L.S. (1978). Mind in society: The development of higher psychological processes. Cambridge, MA: Harvard University Press.
Yelland, N. \& Masters, J. (2007). Rethinking Scaffolding in the Information Age. Computers \& Education, 48(3), 362-82.
Fecha de recepción: 21-11-2016

Fecha de evaluación: 01-12-2016

Fecha de aceptación: 19-12-2017

-181 -

Píxel-Bit. Revista de Medios y Educación. $N^{o} 51$ Julio 2017. ISSN: 1133-8482. e-ISSN: 2171-7966. doi: http://dx.doi.org/10.12795/pixelbit.2017.i51.11 